\section{Associações entre características de organizações híbridas e elementos contextuais de resiliência organizacional}

\author{
Hélida Norato ${ }^{1}$ e Anderson Ribeiro Duarte
}

\section{RESUMO}

Estudos recentes apontam que a gestão de organizações híbridas enfrenta desafios para viabilizar a conciliação de lógicas concorrentes. Sendo assim, este estudo identifica características singulares de organizações híbridas, apresenta um mapa conceitual desenvolvido através de uma estratégia concept-centric para sistematizar novas relações entre dimensões e características sigulares, além de propor um modelo processual que descreve associações entre características singulares de organizações híbridas com elementos contextuais de resiliência organizacional. Foi possível verificar que as características singulares utilizadas no mapa conceitual reforçam processos capazes de contribuir para resiliência organizacional. Os resultados apresentam uma perspectiva de associação dos elementos contextuais da resiliência organizacional com três características de organizações híbridas: (i) missão, benefícios e impactos pretendidos; (ii) impacto social; (iii) governança e parcerias. Detectou-se nessas características um potencial desenvolvedor de dimensões capazes de auxiliar para resiliência organizacional, assim como um forte senso de propósito. Ademais, valores fundamentais como integração, interdependência e cooperação ficaram evidentes dentre as dimensões sob investigação.

Palavras-chave: organizações híbridas; resiliência organizacional; lógicas concorrentes

\section{Associations between characteristics of hybrid organizations and contextual elements of organizational resilience}

\section{ABSTRACT}

Recent studies point out that the management of hybrid organizations faces the challenge of facilitating the conciliation of competing logics. This paper identifies unique characteristics of hybrid organizations and presents a conceptual map, which was elaborated through a concept-centric strategy to systematize new relationships between dimensions and unique characteristics. We also propose a processual approach that describes associations between unique characteristics of hybrid organizations with contextual elements of organizational resilience. The results present a perspective of associating the contextual elements of organizational resilience with three characteristics of hybrid organizations: (i) Mission, benefits and intended impacts; (ii) social impact; (iii) governance and partnerships. In these characteristics, a potential for the development of dimensions capable of assisting the organizational resilience was detected. A strong sense of purpose and core values; integration; interdependence and cooperation are very evident among the dimensions under investigation.

\section{Keywords: hybrid organizations; organizational resilience; competing logics}

1 Esta pesquisa foi apoiada pela Coordenação de Aperfeiçoamento de Pessoal de Nível Superior (CAPES) e também pela Universidade Federal de Ouro Preto (UFOP).

Recebido em: 22/01/2019 Revisado em: 02/04/2019 Aprovado em: 23/01/2020

Check for updates

Hélida Norato (iD,

Universidade Federal de Ouro Preto, Brasil

Doutora em Administração pela Fundação Getúlio Vargas, São Paulo, Brasil

helida@ufop.edu.br

Anderson Ribeiro Duarte (iD,

Universidade Federal de Ouro Preto, Brasil

Doutor em Estatística pela

Universidade Federal de Minas Gerais, Brasil

anderson.duarte@ufop.edu.br 


\section{Introdução}

A literatura sobre organizações apresenta atenção crescente sobre organizações híbridas e resiliência organizacional. As organizações híbridas, em sua maioria, enfrentam dificuldades na tentativa de conciliação de lógicas concorrentes. Por essa razão, a busca por características de organizações híbridas capazes de favorecer a conciliação de lógicas concorrentes é de grande relevância nessa área de estudo. Ademais, a existência de uma lacuna no desenvolvimento de um diálogo entre características de organizações híbridas e resiliência organizacional estabelece um desafiador objeto de investigação. Além disso, a crescente representatividade de organizações que promovem a geração de valor social de forma lucrativa ilustra a possibilidade dessa direção de investigação.

O termo "organização híbrida" é utilizado para designar organizações que atrelam simultaneamente foco na criação de valor social e financeiro (Battilana, Lee, Walker, \& Dorsey, 2012). O contexto de organizações híbridas está circundado de várias lógicas institucionais e algumas possuem orientações distintas. Ainda nessa contextualização, "lógicas concorrentes" decorrem da disparidade de orientações exteriorizadas do foco social e financeiro (Doherty, Haugh, \& Lyon, 2014). O termo "resiliência organizacional" representa a capacidade das organizações em gerar ações próprias e singulares para cenários desafiadores e adversos (Lengnick-Hall, Beck, \& Lengnick-Hall, 2011).

Sendo assim, este estudo explora o contexto específico de organizações híbridas sobre a perspectiva de resiliência organizacional. Além disso, motiva a proposição de um diálogo direcionado através de características das organizações híbridas, que envolvem processos capazes de promover a resiliência organizacional. Essa discussão é pouco explorada na literatura, portanto, uma abordagem específica pode fornecer elementos para que as organizações possam desenvolver novas capacidades que contribuam para a sua sustentabilidade no mercado.

A concepção de um estudo baseado na proposição do referido diálogo estabelece o enquadramento de inserção da seguinte questão de pesquisa: quais as principais características das organizações híbridas que envolvem processos capazes de promover a resiliência organizacional? Dessa questão são estabelecidos os seguintes objetivos diretos dessa investigação: identificar características de organizações híbridas que reforçam processos capazes de contribuir para a resiliência organizacional; produzir um mapa conceitual baseado nas características identificadas; identificar associações entre características; e elementos contextuais de resiliência organizacional necessários para construir um modelo processual.

Inicialmente, este estudo elenca características capazes de favorecer a conciliação de lógicas concorrentes através de revisão de literatura. Posteriormente, estabelece associações entre algumas dessas características com elementos contextuais de resiliência organizacional. Logo, essas duas etapas permitem a elaboração de um mapa conceitual representativo das associações estabelecidas. Por fim, um modelo processual, que detalha as referidas associações, é apresentado. Pode-se afirmar que a presente 
investigação direciona para interseções entre o campo de organizações híbridas e resiliência organizacional, além de favorecer uma agenda entre estes dois domínios.

Entre os desenvolvimentos de pesquisa no campo de organizações híbridas algumas abordagens (Pache \& Santos, 2013; Mair, Mayer, \& Lutz, 2015) convergem para a análise do potencial para equilibrar lógicas concorrentes. Tais abordagens adotam perspectivas de governança organizacional, do desacoplamento e de comprometimento organizacional.

Sendo assim, este estudo gera recursos representativos diretos para: (i) pesquisadores engajados na compreensão de dimensões da gestão de organizações híbridas e (ii) organizações com propósito de promover impacto social. Além disso, gera também recursos indiretos para (iii) gestores quanto às perspectivas de gestão que podem ampliar a resiliência organizacional.

O artigo está estruturado da seguinte forma: A próxima seção apresenta bases teóricas relevantes para a investigação; posteriormente, temos os procedimentos metodológicos empregados para o desenvolvimento do estudo; a exposição dos resultados; e o relato das características singulares de organizações híbridas. Através de um mapa conceitual são estabelecidas associações entre essas características e a resiliência organizacional, e um modelo processual descreve as associações estabelecidas. Por fim, são apresentadas conclusões com implicações teóricas e propostas de continuidade com sugestões de estudos empíricos futuros.

\section{- Base Teórica}

A discussão de assuntos desse propósito exige a exposição de conceitos de termos relevantes como organizações híbridas, lógicas concorrentes e resiliência organizacional. Pesquisadores da área, em geral, possuem clareza acerca de tais conceitos. Por outro lado, pesquisadores de demais áreas podem precisar de uma delimitação mais clara.

\section{Organizações híbridas}

O termo "organização híbrida" é mencionado em pesquisas de gestão pública e de organizações sem fins lucrativos a partir do início do século XXI (Wood Jr., 2010). Esse termo, em geral, refere-se a organizações que operam na interface entre o setor público e privado (Pache \& Santos, 2013; Ávila \& Amorim, 2017). A transposição de limites entre esses setores é destacada por Battilana, Besharov e Mitzinneck (2017) como efeito da pluralidade dos modelos híbridos.

Em decorrência dessa pluralidade, o trabalho de Battilana et al. (2017) propôs três abordagens amplas para análises pertinentes ao termo. Cada uma dessas abordagens tem seu próprio enfoque, sendo: (i) identidades; (ii) formatos; (iii) racionalidades. Portanto, tal proposição representa um norte para novos estudos neste campo.

Ainda frente a essa pluralidade, vários autores afirmam que o termo "organização híbrida" é utilizado com intuito de designar organizações que 
atrelam simultaneamente o foco na criação de valor social e econômico (Battilana et al., 2012; Ebrahim, Battilana \& Mair, 2014; Mair et al., 2015; Mcmullen \& Warnick, 2016; Kannothra, Manning, \& Haigh, 2018; Moss, Renko, Block, \& Meyskens, 2018; Alexius \& Furusten, 2019; Bauwens, Huybrechts, \& Dufays, 2019).

Este estudo aborda organizações híbridas através do enfoque de racionalidades, que segundo Battilana et al. (2017), envolve modelos e práticas que orientam a forma de interação com ambiente. Para Battilana et al. (2017), as racionalidades moldam objetivos que guiam as organizações ao se envolverem em dupla missão (social/financeira). Em virtude dessa missão dual, as organizações híbridas buscam a associação inconvencional de elementos que orientam a interação com o ambiente (Pache \& Santos, 2013; Battilana et al., 2017).

Portanto, este estudo considera como organização híbrida aquela que persegue uma missão dupla (social/financeira), sem desconsiderar as diversidades dessas organizações que podem estar associadas às racionalidades identidades ou formas.

\section{Lógicas concorrentes}

A literatura relativa às organizações híbridas apresenta crescente fluxo que discute a noção de lógicas concorrentes neste campo. Uma das principais origens dessa discussão é o trabalho de Pache e Santos (2010), que originou importante contribuição para a teoria institucional. O foco simultâneo na criação de valor social e financeiro é destacado por Pache e Santos (2010) como um dos mecanismos que ampliam a ocorrência de demandas institucionais conflitantes.

Estudos analisam as tensões vivenciadas por organizações híbridas e tais análises decorrem da associação de elementos compostos de orientações dispares que são provenientes de diferentes lógicas institucionais (Battilana \& Dorado, 2010; Pache \& Santos, 2013; Doherty et al., 2014; Santos et al., 2015; Kannothra et al., 2018). Esses estudos abordam lógicas concorrentes em relação às dualidades de gestão vivenciadas no ambiente organizacional. Tais dualidades decorrem da orientação para práticas sociais e de mercado.

Poucos estudos apresentam argumentos para explicar a conciliação de lógicas concorrentes. O trabalho de Pache e Santos (2013) desenvolve explicação para a conciliação através de perspectivas de desacoplamento e comprometimento organizacional. O estudo de Mair et al., (2015) adota a perspectiva de governança organizacional para explicar a conciliação.

Segundo Doherty et al. (2014), a tensão vivenciada por organizações híbridas entre orientações sociais e de mercado, e entre múltiplos stakeholders como funcionários e voluntários, denota a manifestação de lógicas concorrentes nessas organizações. Sendo assim, as organizações híbridas desenvolvem uma capacidade singular para elaborar e executar soluções inovadoras para gerar impacto social (Battilana et al., 2015). O impacto gerado é ressaltado por Martin e Osberg (2007), como a promoção de benefícios que contribuem para alguma parcela desfavorecida da 
população. De acordo com Kroeger \& Weber (2014), na busca por promover impacto social, as organizações híbridas atuam em ambientes adversos e desafiadores.

\section{Resiliência organizacional}

A resiliência organizacional é considerada por Lengnick-Hall et al. (2011) como um conhecimento processual. Através desse conhecimento, uma organização pode gerar ações próprias e distintas para circunstâncias desafiadoras e adversas. Isso ocorre através de uma sistemática que promove o desenvolvimento de novas capacidades e que contribui para a sustentabilidade da organização no mercado.

O estudo seminal que apresentou o conceito de resiliência no campo de gestão foi desenvolvido por Meyer (1982) e o trabalho de Linnenluecke (2017) confirma essa afirmação. A perspectiva do estudo de Meyer (1982) relacionou o conceito como uma variável explicativa e de resultado, capaz de identificar tempo de resposta e condições necessárias para restabelecer os padrões normais de processos organizacionais que foram alterados por eventos externos (Lengnick-Hall \& Beck, 2016; Linnenluecke, 2017).

Em sentido oposto à proposta original de resiliência no campo de gestão, o conceito passou a ser analisado em razão de eventos no ambiente interno das organizações. A partir dos anos noventa do século XX, acidentes ocorridos em ambientes internos que impactaram negativamente nas dimensões econômicas, sociais e ambientais contribuíram para novas perspectivas sobre o conceito de resiliência no campo de gestão (Linnenluecke, 2017). Essas novas perspectivas estavam fundamentalmente embasadas em conceitos de confiabilidade e visão baseada em recursos (Danes et al., 2009; Bergheim et al., 2013; Memili, Welsh, \& Luthans, 2013).

Contudo, um dos marcos de difusão de estudos sobre resiliência no campo de gestão foi o ataque terrorista de 11 de setembro de 2001, que reafirmou o prisma de análise do conceito em relação aos eventos externos capazes de alterar significativamente processos organizacionais (Coutu, 2002; Sutcliffe \& Vogus, 2003; Gittell, Cameron, Lim \& Rivas, 2006; Somers, 2009). As novidades de perspectivas que surgiram após o ataque foram fundamentadas a partir das competências dos funcionários, com enfoques originados da psicologia, que atribuem na resiliência a crença positiva no poder de recuperação (Luthans, 2002; Avey, Reichard, Luthans, \& Mhatre, 2011; Shin et al., 2012; Luthans, Luthans, \& Avey, 2014). Vários trabalhos como os de Vogus e Sutcliffe (2007), Abdullah, Noor e Ibrahim, (2013) e Korber e McNaughton (2018) identificam que dois estudos destacam-se nesse marco de difusão da pesquisa sobre resiliência organizacional: o trabalho de Sutcliffe e Vogus (2003) e o trabalho de Lengnick-Hall et al. (2011).

As fronteiras da resiliência organizacional estão delimitadas dentro de três principais fluxos relacionados aos eventos externos, aos incidentes internos ou a ambos, de modo a caracterizar potencial de sustentabilidade (Lengnick-Hall \& Beck, 2016; Linnenluecke, 2017; Korber \& McNaughton, 2018). A partir desses fluxos, as principais correntes de pesquisa de resiliência 
organizacional foram desenvolvidas em torno das seguintes abordagens: (i) capacidade de reação e solução da organização aos fatores externos; (ii) níveis de confiança; (iii) competências da equipe interna; (iv) flexibilidade de modelos de negócios e (v) minimização de pontos vulneráveis através princípios de gestão da cadeia de suprimentos (Linnenluecke, 2017).

\section{Drocedimentos Metodológicos}

\section{Revisão sistemática para identificação de características singulares}

A delimitação dos conceitos anteriores leva ao interesse de identificar características de organizações híbridas. A revisão realizada para delimitar tais conceitos deixa clara a ausência de pesquisas capazes de reconhecer pontualmente tais características. A condução de uma revisão sistemática foi necessária devido à escassez de estudos que abordam de forma específica tais características de organizações híbridas através da perspectiva de resiliência organizacional.

Pesquisadoresnecessitam demetodologiaeficaz parabuscarinformações na literatura e trazer reconhecimento aos seus estudos. Procedimentos mais ingênuos de revisão, em geral, fornecem resultados controversos. Sendo assim, para ampliar a credibilidade, a revisão foi conduzida seguindo uma adaptação de passos básicos da estratégia de revisão sistemática.

Uma revisão sistemática parte da premissa de responder uma pergunta formulada de forma clara e utiliza de métodos sistematizados para o procedimento de identificação, seleção e avaliação crítica das obras analisadas (Clarke \& Oxman, 2001).

Diversos autores abordam detalhes sobre o adequado processo de execução de revisões sistemáticas (Okoli \& Schabram, 2010; Uman, 2011; Bearman \& Dawson; 2013). Em particular, foram seguidas as orientações de Okoli e Schabram (2010) para revisões desse tipo. Algumas adaptações foram incluídas para o propósito específico deste estudo. A adoção dessa abordagem contribuiu para integrar e sintetizar a produção acadêmica na área sob investigação, além de proporcionar uma análise crítica do atual estágio do conceito de organizações híbridas.

Okoli e Schabram (2010) fornecem uma adaptação da clássica definição de Fink (2005) para revisão sistemática. Essa adaptação tem interesse na área de Sistemas de Informação e se mostrou adequada para esse estudo. De acordo com Okoli e Schabram (2010), os passos básicos de execução são: (1) o propósito; (2) protocolo e treinamento; (3) busca na literatura; (4) triagem de inclusão; (5) triagem de exclusão; (6) extração de dados; (7) síntese de estudos e (8) escrita da revisão.

O propósito da revisão é obviamente o primeiro passo em qualquer concepção de técnica de revisão de literatura. Neste estudo em particular, o alvo foi identificar as características singulares de organizações híbridas, sobretudo aquelas que reforçam processos capazes de contribuir para 
resiliência organizacional. Essas características serão de grande valia para o alcance dos objetivos de construção do mapa conceitual e do modelo processual já mencionados anteriormente. Quanto ao protocolo, este estudo contou com o envolvimento de apenas dois revisores, o que facilitou grandemente a proposição de um protocolo específico de trabalho.

A estratégia de busca é de grande relevância, sendo necessário deixar explícito todos os detalhes da técnica de busca. Além disso, a segurança de que a pesquisa foi abrangente precisa estar justificada. $O$ fato de 0 conceito de organizações híbridas ser proeminente e ainda estar em construção influenciou de forma direta os procedimentos para a revisão da literatura. Para a literatura sobre organizações híbridas, as publicações para inclusão nesta revisão foram identificadas através de pesquisas na base de dados Scopus. Os critérios que orientaram a escolha dentre as possíveis bases foram a representatividade no meio acadêmico e a disponibilidade de acesso.

Já em relação à abrangência da busca, dois estudos em particular foram adotados como base de orientação para o desenvolvimento da revisão de literatura: os trabalhos de Webster e Watson (2002) e de Creswell (2013). Esses autores afirmam que para garantir a abrangência de uma revisão de literatura, algumas ações são fundamentais: (i) buscar uma narrativa histórica dos temas ou conceitos articulados na pesquisa; (ii) identificar as principais correntes teóricas; e (iii) identificar como a proposta de questão para a pesquisa se insere no contexto da literatura atual. Essas ações foram adotadas para desenvolver a busca de literatura acerca dos conceitos e características de organizações híbridas.

Além disso, no procedimento de seleção de artigos, foram adotados os quatro eixos de avaliação de relevância de artigos apresentados por Treinta, Farias Filho, Sant'Anna e Rabelo (2014). Esses eixos são: (i) o artigo - que envolve uma análise de significância e representatividade do artigo para desenvolvimento do campo; (ii) os autores - que concerne identificar a expressividade desses para a consolidação e evolução dos conceitos no campo; (iii) o jornal - que envolve uma avaliação da visibilidade da produção científica no campo inclusive através de análise de fator de impacto do periódico; e (iv) o tema - que tange uma análise de alinhamento das discussões e conceitos do artigo selecionado com a questão proposta para este estudo. A adoção desses eixos justifica-se também por demonstrar certo alinhamento com as ações praticadas na direção de garantir a abrangência da revisão.

O procedimento de inclusão trabalha em associação com os mecanismos de busca. A triagem de exclusão é o mecanismo de avaliação da qualidade e os critérios de julgamento de trabalhos que tenham qualidade insuficiente para serem incluídos devem ser explicitados. Realizou-se uma pesquisa para publicações com o termo "organizações híbridas" (hybrid organizations). Para manter delimitações do escopo e o foco em gestão e estudos organizacionais, restringiu-se às publicações categorizadas na base de dados na área "Negócios, Gestão e Contabilidade".

A busca forneceu em 458 referências de trabalhos que foram analisadas pelos dois revisores. Inicialmente foram examinados os resumos de todas as 
obras. No primeiro momento, o interesse era verificar o seu alinhamento ao tema. Em situações que o resumo não era suficientemente informativo para a verificação inicial, o artigo era avaliado na sua integralidade. Trabalhos que não revelavam alinhamento com o tema já foram excluídos nessa primeira etapa. Uma análise de consonância das discussões e conceitos da referência selecionada com o assunto deste estudo representa tal alinhamento. Para referências nas quais um dos revisores discordava quanto à exclusão, os artigos foram novamente analisados na íntegra, em busca de uma decisão consensual.

Os critérios de julgamento de trabalhos que não apresentavam alinhamento para serem incluídos foram classificados de acordo com o enquadramento específico e com os conteúdos pertinentes dos trabalhos. Foram excluídas 414 referências cujo enquadramento não estava alinhado com tema em estudo, uma vez que abordavam temas como: alianças estratégicas, ativismo social, condições de trabalho, cooperativas, dicotomia entre estatal e privado, espaços híbridos, estrutura de capital, filantropia, formação profissional, gênero, indicadores de desempenho, inovação tecnológica, investimento social, liderança e poder, mídias sociais, outros tipos híbridos, políticas públicas, problemas sociais, princípios legais, responsabilidade social corporativa, sem fins lucrativos, subsídios, teoria de stakeholders, terceiro setor, triple botton line e transferência de conhecimento. A lista com referências excluídas do estudo esta disponível como material suplementar deste artigo.

A tarefa de extração de dados é direcionada pelo propósito da revisão e as informações efetivamente aplicáveis ao estudo foram extraídas pelos revisores. Em particular, esse procedimento delimitou o conjunto de características singulares de organizações híbridas que é apresentado na seção de análise de resultados.

\section{Síntese de informações de características singulares de organizações híbridas}

A síntese de estudos faz a combinação entre as informações extraídas das obras eleitas para inclusão. Neste estudo, essa análise foi o procedimento para elencar características singulares de organizações híbridas. Essas características estão descritas no Quadro 1, que é apresentado na seção de análise de resultados. Existe alguma subjetividade na delimitação dessas características, ou seja, do ponto de vista deste estudo, acredita-se ter estabelecido o conjunto de características singulares para organizações híbridas capazes de contribuir para resiliência organizacional. Isto, por outro lado, não impede que outras visões possam estabelecer alguma outra característica que este trabalho não elege nesse rol.

A produção do referido quadro de características foi um suporte importante para a elaboração de um mapa conceitual. A finalidade do mapa foi estruturar e avaliar adequadamente as informações obtidas. Sendo assim, este estudo adotou a perspectiva de mapa conceitual fundamentada na teoria de aprendizagem e na teoria do conhecimento (Cañas \& Novak, 2008). Diante disso, considera um mapa conceitual como um elemento gráfico que sistematiza o conhecimento através da relação de informações que adquirem significado com base no fundamento das dimensões de conceitos consolidados (Novak, 1990; Plotnick, 1997; Cañas, Novak \& Reiska, 2015). 
Mapa conceitual e modelo processual

Um mapa conceitual pode ser empregado para representar a proposição de novas relações entre dimensões de conceitos e estimular novos desenvolvimentos sobre algum tema (Cañas et al., 2015). Um mapa conceitual consiste numa abordagem lógica que apresenta relações entre dimensões de conceitos sobre a perspectiva do idealizador (Webster \& Watson, 2002). O mapa conceitual que será apresentado utiliza estratégia concept-centric para estruturar e analisar informações.

A proposta de elaboração de um modelo processual está fundamentada na flexibilidade interpretativa desse tipo de modelo e sua relação com os processos de mudança organizacional. A perspectiva processual é útil para analisar tais processos, levando em consideração a complexidade do desenvolvimento da resiliência organizacional. Um modelo processual é profícuo para compreensão de fenômenos organizacionais relacionados à mudança, pois proporciona uma visão holística de tais fenômenos (Burnes, 2004; Pettigrew, Woodman, \& Cameron, 2001; Dawson, 2005). Processos nessa abordagem referem-se à sequência de ações e relações que se desenvolvem no ambiente organizacional (Pettigrew et al., 2001).

Um modelo processual está centrado no reconhecimento de correlações dinâmicas entre conceitos que oportuniza investigações e aplicações (Bobsin, Petrini \& Pozzebon, 2019). O modelo processual revela-se adequado para explicar a associação de elementos contextuais de resiliência organizacional com características de organizações híbridas devido ao seu caráter holístico. Essa abordagem permite ressaltar um potencial com dimensões que auxiliam para a resiliência organizacional.

\section{Análise de Resultados}

A realização do procedimento de revisão sistemática, descrito na seção anterior, forneceu uma lista de características singulares das organizações híbridas, que será apresentada e discutida na subseção Características singulares das organizações híbridas. Uma síntese com dimensões dessas características que reforçam processos capazes de contribuir para a resiliência organizacional foi elaborada através de um mapa conceitual apresentado na subseção Mapa conceitual. Por fim, associações entre algumas dessas características com elementos contextuais da resiliência organizacional são identificadas e ilustradas através de um modelo processual apresentado na subseção Modelo processual para associação com elementos contextuais de resiliência organizacional.

\section{Características singulares das organizações híbridas}

A identificação de características representa um conjunto de diretivas na conceitualização de organizações híbridas. O Quadro 1 apresenta uma proposta de rol de características singulares, baseada nos argumentos obtidos através da revisão de literatura executada. 
Quadro 1. Características singulares de organizações híbridas.

\begin{tabular}{|c|c|}
\hline Características & Origem \\
\hline $\begin{array}{l}\text { A missão, os benefícios e os } \\
\text { impactos pretendidos }\end{array}$ & $\begin{array}{l}\text { Haigh et al. (2015); Lee \& Jay (2015); Santos et al. (2015); Schmitz (2015); Hahn \& Ince (2016); } \\
\text { Kolk \& Lenfant, (2016); Mcmullen \& Warnick (2016);York et al. (2016b);Zahra \& Wright (2016); } \\
\text { Chandra \& Shang (2017); Manning et al.(2017); Bauwens et al.,(2019); Wolf \& Mair (2019) }\end{array}$ \\
\hline Flexibilidade & Pache \& Santos (2013); Kannothra et al. (2018); Manning et al.(2017) \\
\hline Governança e Parcerias & $\begin{array}{l}\text { Mitronen \& Möller (2003); Ebrahim et al. (2014); Anheier \& Krlev (2015); Haigh et al. (2015); Lee \& } \\
\text { Jay (2015); Grossi, \& Thomasson (2015); Mair et al. (2015); Schmitz (2015); Hahn \& Ince (2016); } \\
\text { Kolk \& Lenfant, (2016); Alberti \& Varon Garrido (2017); Wolf \& Mair (2019) }\end{array}$ \\
\hline Impacto social & $\begin{array}{l}\text { Barki et al. (2013); Jäger, \& Schröer (2014); Haigh et al. (2015); Santos et al. (2015); Manning } \\
\text { et al. (2017) }\end{array}$ \\
\hline Inovação & $\begin{array}{l}\text { Barki et al. (2013); Jay (2013); Parra (2013); Wilson \& Post (2013); Lee \& Jay (2015); Mair et al. } \\
\text { (2015); Alberti \& Varon Garrido (2017); Almandoz et al.(2017); Mongelli \& Rullani (2017) }\end{array}$ \\
\hline Múltiplas formas organizacionais & $\begin{array}{l}\text { Koppell (2003); Pache \& Santos (2013); Battilana \& Lee (2014); Anheier \& Krlev (2015); Doherty } \\
\text { et al. (2014); Mair et al. (2015); Almandoz et al.(2017); Battilana (2018); McMullen (2018); Park } \\
\text { (2019); Reissner (2019). }\end{array}$ \\
\hline $\begin{array}{l}\text { Relações orientadas para } \\
\text { sustentabilidade }\end{array}$ & $\begin{array}{l}\text { Battilana \& Dorado, (2010);:Parra (2013); Battilana et al. (2015); Lee \& Jay (2015); York et al. } \\
\text { (2016b); Zahra \& Wright (2016); Alberti \& Varon Garrido (2017); Ávila \& Amorin, (2017); Mongelli } \\
\text { et al.(2017); Alexius \& Furusten (2019); Wolf \& Mair (2019) }\end{array}$ \\
\hline Tensão de recursos & $\begin{array}{l}\text { Mitronen \& Möller (2003); Pache (2010); Pache \& Santos (2013); Mair et al. (2015); Johansen et al., } \\
\text { (2015); Dufays \& Huybrechts (2016); York et al.(2016a); Chandra \& Shang (2017); Roundy (2017); } \\
\text { Schildt \& Perkmann (2017); Battilana (2018); Park (2019); Reissner (2019). }\end{array}$ \\
\hline
\end{tabular}

Fonte: Elaboração própria.

A missão, os benefícios e os impactos pretendidos representam uma única característica que contribuí para a distinção das organizações híbridas no ambiente empresarial. Essa característica impulsiona o desenvolvimento de atividades que promovem impacto social (Doherty et al., 2014; Ebrahim et al., 2014). Um elemento norteador dos processos e práticas organizacionais é representado por essa característica, que estimula inquietação na busca por novas formas de atuação. Para as organizações híbridas, essas novas formas devem ser capazes de promover transformações na vida das pessoas expostas às atividades da organização e ainda, garantir a sustentabilidade da organização no mercado (Barki, Izzo, Torres, \& Aguiar, 2013).

A flexibilidade representa uma característica inerente às organizações híbridas. Elas são capazes de mesclar formatos e práticas organizacionais convencionais na busca por equilibrar lógicas sociais e de mercado (Pache \& Santos, 2013; Kannothra et al., 2018). As organizações híbridas conseguem agregar propriedades de múltiplos setores (Pache \& Santos, 2013). Além disso, a versatilidade das organizações híbridas se reflete no potencial para desenvolver modelos de negócios mercadologicamente e financeiramente sustentáveis e que sejam capazes de promover transformações positivas na vida das pessoas expostas às atividades da organização (Santos et al., 2015). A viabilidade dos modelos de negócios é baseada em uma prática de gestão que estimula um processo interativo entre os stakeholders e também o desenvolvimento de redes colaborativas. 
Mair et al. (2015) defendem a existência de alinhamento da prática de gestão de organizações híbridas com orientações e definições da literatura sobre governança corporativa. A governança desempenha papel fundamental para que as organizações híbridas desenvolvam competências para evitar que a geração de valor social seja colocada em segundo plano frente à geração de valor financeiro (Ebrahim et al., 2014). Organizações híbridas usufruem do conhecimento coletivo que transita de forma livre entre os stakeholders e representam importante fonte de geração de valor para os negócios (Hahn \& Ince, 2016). A adoção efetiva de práticas de governança proporciona terreno fértil para interações favoráveis com os stakeholders que fomentam a geração de valor (Lee \& Jay, 2015).

O impacto social representa uma característica que traduz uma das faces do objetivo dual das organizações híbridas: a geração de valor social de forma sustentável. A busca pela promoção de impacto social é perseguida nessas organizações através de métodos e abordagens de negócios que geram soluções integrativas para problemas sociais (Miller, 2012; Wilson \& Post, 2013). Ademais, o impacto social corresponde à geração de valor social positivo. Conforme Martin e Osberg (2007), organizações híbridas são singulares por gerar valor na forma de "benefício transformacional"; em outras palavras, benefícios capazes de contribuir para alterar alguma condição desfavorecida de uma parte da população. Kroeger e Weber (2014) destacam que o prelúdio da geração de valor social é a realização de uma intervenção social relevante para o bem-estar da população desfavorecida. Esses autores adotam o bem-estar no sentido de julgamento interno dos indivíduos beneficiados. O nível de geração de valor proporcionado por organizações híbridas também está relacionado com o principal mercado de atuação dessas organizações: o mercado que envolve consumidores que estão na base da pirâmide. Esses consumidores não dispõem facilmente de recursos para realizar aquisições, não têm oportunidades claras junto às ofertas tradicionais, ou têm dificuldades de acessá-las, e ainda, não percebem facilmente o valor agregado dos produtos (Gupta, Beninger, \& Ganesh, 2015; Santos et al., 2015; Quinn \& Munir, 2017).

Focadas em algum tipo de intervenção social relevante, as organizações híbridas desenvolvem soluções inovadoras para problemas sociais (Lepoutre, Justo, Terjesen, \& Bosma, 2013; Barki et al., 2013; Choi \& Majumdar, 2014). A inovação representa uma importante característica das organizações híbridas e viabiliza a transposição de barreiras de transação. Através da inovação em modelos de negócios, as organizações híbridas viabilizam transações em mercados nas quais transações de empresas convencionais podem não se efetivar por empecilhos diversos, como dificuldade de acesso, por exemplo (Santos et al., 2015). As organizações híbridas inovam ao mesclarem formatos de gestão para promover geração de impacto social de forma sustentável.

A capacidade de mesclar diversos formatos de gestão revela uma das características mais singulares das organizações híbridas, que aqui é denominada por múltiplas formas organizacionais. Organizações híbridas são capazes de romper os limites entre arranjos institucionais vigentes. Organizações híbridas não se enquadram nos setores públicos, privado ou sem fins lucrativos (Mair et al., 2015). As organizações híbridas não se 
restringem aos modelos de negócios que buscam mesclar geração de valor social e financeiro. Para além desse composto, organizações híbridas realizam o mix entre modelos de gestão institucionalizados (Pache \& Santos, 2013). Dessa forma, as organizações híbridas utilizam da capacidade de mesclar diversos formatos de gestão (múltiplas formas organizacionais) para moldá-las em prol de objetivos orientados para a sustentabilidade conforme as demandas as quais são expostas.

Diversos processos em toda a cadeia de suprimentos do negócio revelam relações orientadas para a sustentabilidade. Um exemplo disso é a preocupação no envolvimento de trabalho inclusivo, logística reversa, insumos orgânicos (Lee \& Jay 2015). Através de ações sustentáveis, as organizações desse tipo são propensas a desenvolver parcerias em que a colaboração é o elemento chave para o alcance dos resultados, além do envolvimento dos stakeholders guiado por valores que reforçam práticas sustentáveis (Haigh et al., 2015; Lee \& Jay, 2015). O potencial para desenvolvimento de uma rede colaborativa revela-se um importante elemento para que essas organizações desenvolvam competências para lidar com a tensão entre recursos.

Por fim, elenca-se a tensão de recursos como característica das organizações híbridas. Essa tensão é principalmente experimentada por organizações desse tipo, em face do risco iminente de se desviarem da sua missão social, em detrimento da manutenção de sua sustentabilidade financeira (Santos et al., 2015). As organizações híbridas desenvolvem uma capacidade particular para equilibrar lógicas de mercado e sociais, além de promoverem habilidades para equacionar a oscilação de recursos tangíveis (Pache \& Santos, 2013; Krueger \& Behringer, 2017).

\section{Mapa conceitual}

A proposta de construir um mapa conceitual é a de sistematizar novas relações entre dimensões e conceitos. As características singulares de organizações híbridas elencadas no Quadro 1 serão sistematizadas através de um mapa conceitual. A ausência de uma clara delimitação de características singulares de organizações híbridas justifica a necessidade de sistematizar tais características. A produção do mapa conceitual serviu ainda para identificar dimensões dessas características que reforçam processos capazes de contribuir para a resiliência organizacional.

Além disso, o processo de construção do mapa conceitual envolveu a busca por significados com base nas três dimensões da resiliência organizacional: (i) elementos cognitivos; (ii) elementos comportamentais e (iii) elementos contextuais (Lengnick-Hall et al., 2011). Essa busca por significados destacou elementos contextuais da resiliência organizacional como conceitos propícios para estimular novos desenvolvimentos sobre características de organizações híbridas que envolvem processos capazes de promover a resiliência organizacional.

Os procedimentos descritos na construção do mapa conceitual resultaram em um diagrama que representa a proposta de inéditas relações entre características singulares de organizações híbridas e elementos contextuais da resiliência organizacional. Além disso, foi possível estabelecer 
conexões associativas com o conceito de resiliência organizacional, as quais estão ilustradas por meio do mapa conceitual apresentado na Figura 1.

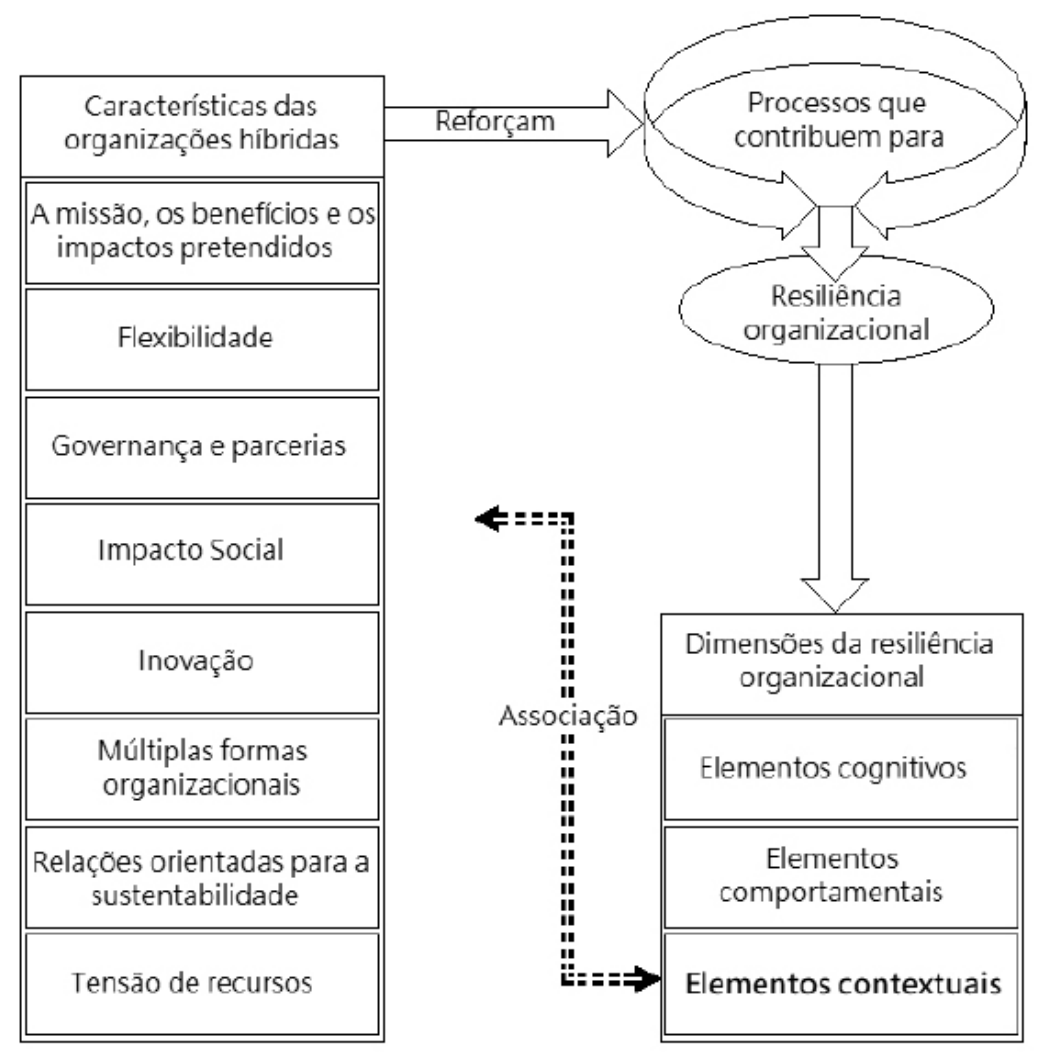

Figura 1. Mapa conceitual.

Fonte: Elaboração própria.

O conjunto de características singulares identificado apoia a conciliação de lógicas concorrentes pelas organizações híbridas. Isso ocorre no contexto do desenvolvimento de processos que promovem a resiliência organizacional. A conciliação se manifesta, por exemplo, em esforços empreendidos para equacionar a geração de impacto social com a geração de retorno financeiro e de mercado. Nesse cenário, essas organizações convivem com variadas barreiras de transação. Para transpor tais barreiras, as organizações híbridas envolvem-se em uma dinâmica de adequação, conciliação e aprendizado, e tal dinâmica promove novos potenciais de gestão. Por conseguinte, este estudo apresenta uma abordagem para explicar a concililiação de lógicas concorrentes e as bases dessa abordagem são as características das organizações hibridas que envolvem processos capazes de promover a resiliência organizacional. Uma síntese com as características das organizações hibridas e os respectivos processos capazes de contribuir para resiliência organizacional pode ser visualizada no Quadro 2. 
Quadro 2. Associações entre características de organização híbrida e resiliência organizacional.

\begin{tabular}{ll}
\hline \multicolumn{1}{c}{ Característica } & \multicolumn{1}{c}{ Processos capazes de contribuir para resiliência organizacional } \\
\hline $\begin{array}{l}\text { A missão, os benefícios e os impactos } \\
\text { pretendidos. }\end{array}$ & Função explícita de promover transformações positivas. \\
Flexibilidade & Diretrizes para harmonizar e moldar processos de gestão. \\
Governança e Parcerias & Prática de gestão participativa desenvolvimento de redes colaborativas. \\
Impacto social & Modelos de negócios para transposição de barreiras de transação. \\
Inovação & Gestão plural estratégias inéditas. \\
Múltiplas formas organizacionais & Diversificadas configurações organizacionais. \\
Relações orientadas para a sustentabilidade & Valores que reforçam práticas sustentáveis. \\
Tensão de recursos & Potencial para equacionar a oscilação de recursos tangíveis e incompatibilidades com \\
\hline
\end{tabular}

Fonte: Elaboração própria.

A literatura sobre resiliência organizacional apresenta diversas análises para definir a capacidade de uma organização resiliente (Mallak, 1998; Kendra \& Wachtendorf, 2003; Lengnick-Hall \& Beck, 2005; McManus, Seville, Brunsdon, \& Vargo, 2007; Woods \& Wreathall, 2008; Gibson \& Tarrant, 2010; Braes \& Brooks, 2011; Lengnick-Hall et al., 2011; Windle, Bennett, \& Noyes, 2011; Lee, Vargo, \& Seville, 2013; Ayala \& Manzano, 2014). Entretanto, a proposta de estabelecer associação entre as capacidades de organizações resilientes e características de organizações híbridas é uma lacuna de pesquisa ainda aberta. É possível descrever, de foma mais detalhada, as constatações que sugerem tais associações para cada uma das características mencionadas no Quadro 2.

A missão, os benefícios e os impactos pretendidos representam uma única característica que envolve estratégias evidentemente centradas em promover transformações positivas na comunidade exposta às atividades da organização. Esse caráter manifesto retrata um sólido senso de propósitos e guia os procedimentos no âmbito organizacional. De acordo com Gibson e Tarrant (2010), os valores organizacionais representam um dos principais drivers de resiliência. O estabelecimento de metas claras, guiadas por valores fundamentais, fortalece o alinhamento organizacional e favorece a promoção de ajustes necessários nos procedimentos internos da organização de modo dinâmico (Vogus \& Sutcliffe, 2007; Braes \& Brooks, 2011). O fortalecimento é construído através de uma rede multidimensional de ações positivas que representam práticas e comportamentos convenientes (Cooper, Estes, \& Allen, 2004; Ayala \& Manzano, 2014). O envolvimento das organizações em atividades transformadoras e disruptivas revela qualidades que fortalecem a organização frente a condições desafiadoras (Braes \& Brooks, 2011; Lengnick-Hall et al., 2011).

A capacidade adaptativa das organizações resilientes pode ser associada com duas características de organizações híbridas, "flexibilidade" e "múltiplas formas organizacionais", as quais habilitam a lidar com variadas configurações organizacionais de forma eficaz. Além disso, promovem um mix único de processos de gestão através do emprego de recursos dinâmicos. 
Essas habilidades revelam dimensões que contribuem de forma efetiva para a construção da resiliência organizacional e tal perspectiva é corroborada por pesquisas que sustentam que a essência da resiliência organizacional está na capacidade de adaptação e contínua geração de respostas singulares às mudanças ambientais (Lengnick-Hall \& Beck, 2005; Weick \& Sutcliffe, 2011; Lee et al., 2013; Ayala \& Manzano, 2014; Limnios, Mazzarol, Ghadouani, \& Schilizzi, 2014).

Alguns estudos destacam que pela governança participativa, organizações híbridas se tornam capazes de responder às demandas sociais de modo efetivo e financeiramente sustentável por meio do envolvimento ativo de stakeholders (Dardour, 2012; Wilson \& Post, 2013; Doherty, et al., 2014). A característica destacada como "governança e parecerias", evidencia a prática de gestão nessas organizações, guiada por princípios de governança corporativa. Essa prática estimula envolvimento da equipe e alinhamento de interesses entre os stakeholders. Ademais, os processos de gestão nas organizações híbridas revelam potencial de contribuição para resiliência, a qual, no contexto da organização, transparece através de ações voltadas para integração, interdependência e cooperação. De acordo com Mallak (1998), Lengnick-Hall et al. (2011) e Rodríguez-Sánchez e Perea (2015), as ações mencionadas anteriormente representam dimensões que estão positivamente associadas à construção da resiliência organizacional.

Os valores de organizações híbridas promovem um propósito comum e reforçam o cunho de geração de impacto. Ações organizacionais são planejadas com intuito de transpor as barreiras de transação que, em muitos casos, circundam as operações no contexto das organizações híbridas (Santos et al., 2015). O impacto social representa resultados alcançados exclusivamente em face das intervenções no ambiente promovidas pela atividade desempenhada por organizações híbridas (Barki et al., 2013). Os modelos de negócios dessas organizações revelam a competência em perseverar frente às adversidades ambientais. Tal perseverança contribui para a construção da resiliência segundo Tedeschi e Calhoun (2004). O impacto social, ou seja, os benefícios transformacionais propiciados à comunidade, é pivô de motivação da equipe e dos processos organizacionais dos híbridos. Para Bullough, Renko e Myatt (2014), as pessoas que confiam em seu potencial e estão determinadas em perseverar para promover benefícios transformacionais na comunidade representam exemplos para construção da resiliência. Rodríguez-Sánchez e Perea (2015) utilizam o conceito de eficácia coletiva de Bandura (1997) para relacionar construção de resiliência com a crença de um grupo em seu potencial para fomentar mudanças positivas na comunidade. A importância do sentimento de grupo e a força de equipe é um elemento discutido em pesquisas sobre resiliência (Mallak, 1998; Somers, 2009; Rodríguez-Sánchez \& Perea; 2015).

A inovação, para as organizações híbridas, representa um processo chave, que sustenta as atividades para a geração de valor social e viabiliza dinâmicas para superar a escassez e tensão de recursos (Lee \& Jay, 2015; Santos et al., 2015). Essas dinâmicas impõem uma revisão contínua do comportamento organizacional. Através de um processo contínuo de reavaliação, a organização desenvolve potencial para se adaptar e 
estabelecer estratégias proativas para reagir às adversidades do mercado (Hamel \& Valikangas, 2004; Sutcliffe \& Vogus 2003).

A relevância do poder de equipe e das práticas organizacionais para organizações resilientes impulsiona outra associação com uma das características de organizações híbridas, aqui nominada de "relações orientadas para sustentabilidade". Essa associação justifica-se porque tal característica propaga ações em que as formas de relacionamento da equipe têm como premissa uma visão global. Formas de relacionamento com visão global dos processos pelos integrantes permitem alcançar a melhoria dos processos e a sustentabilidade. Para Lampel, Bhalla e Jha (2014), a construção da resiliência envolve procedimentos que viabilizam o incremento de competências em prol do reestabelecimento e manutenção da eficiência. Essa visão é corroborada por outros estudos que destacam o desenvolvimento de formas de relacionamentos da equipe e que garantem os processos sustentáveis como elementos para constituição de organizações resilentes (Mallak, 1998; Rodríguez-Sánchez, \& Perea, 2015). Relações orientadas para sustentabilidade envolvem uma sistemática capaz de estruturar uma rede de relacionamentos. Lengnick-Hall et al. (2011) salientam que tal rede viabiliza processos e ativos intangíveis, além de constituírem-se como comportamentos resilentes e valorosos nos processos decisórios e na gestão de recursos.

Quanto à tensão de recursos, os processos adotados para gestão de recursos por organizações desse tipo são estabelecidos para equacionar a oscilação de recursos tangíveis e também para harmonizar os recursos intangíveis em face dessa variação, assim como responder aos desafios circunstanciais. Esses processos revelam dimensões que fomentam resiliência organizacional. Alguns estudos ressaltam como elemento para a construção de resiliência a conciliação de recursos através de configurações inovadoras como uma forma de sobreviver e prosperar em condições ambientais adversas (Mallak, 1998; Woods \& Wreathall, 2008; Ayala \& Manzano, 2014).

\section{Modelo processual para associação com elementos contextuais de resiliência organizacional}

A capacidade de adaptação e a contínua dinâmica de inovação representam habilidades fundamentais para construção da resiliência organizacional (Lengnick-Hall et al., 2011). Por outro lado, essas habilidades não são suficientes para uma organização efetivamente resiliente. Estudos defendem que a construção eficaz da resiliência organizacional envolve um mix de habilidades e processos que orientem todas as atividades organizacionais de modo a desenvolver recursos dinâmicos (Lengnick-Hall \& Beck, 2003, 2005; Lengnick-Hall et al., 2011). Esse mix envolve três dimensões da resiliência organizacional: (i) elementos cognitivos; (ii) elementos comportamentais e (iii) elementos contextuais (Lengnick-Hall et al., 2011).

Este estudo em particular concentrou-se em elementos contextuais da resiliência organizacional, uma vez que tais elementos representam 
competências internas da organização. Além disso, esses elementos viabilizam a interação com agentes externos em prol de atitudes assertivas frente às condições ambientais adversas. O primeiro elemento é a segurança psicológica, que possibilita condições para que os membros da organização se sintam confiantes para desenvolver novas perspectivas e competências, além de assumir riscos (Lengnick-Hall et al., 2011). O segundo elemento é o capital social profundo, que fortalece os elos entre os membros da organização com base em interações respeitáveis. Elos mais fortes viabilizam a transmissão de conhecimentos tácitos como o senso de interdependência e a colaboração, de modo a favorecer a geração de recursos organizacionais coesos e dinâmicos (Lengnick-Hall et al., 2011).

Os dois primeiros elementos contextuais de resiliência organizacional podem ser associados às características das organizações híbridas: "a missão, os benefícios, e os impactos pretendidos" e "impacto social". Essa associação justifica-se porque tais características ressaltam dimensões como "propósito explícito e orientação concisa para transposição de barreiras ambientais". Tais dimensões são capazes de contribuir para a construção da resiliência organizacional através de um forte senso de propósito e valores fundamentais.

O terceiro elemento contextual da resiliência organizacional é o poder e as responsabilidades difusas. Esse elemento possibilita maior comprometimento dos membros da organização e também fomenta o envolvimento de todos na supervisão das atividades com o cuidado necessário com os objetivos organizacionais (Lengnick-Hall et al., 2011). O quarto e último elemento é o acesso à rede de recursos ampla que viabiliza a disponibilidade de parcerias estratégicas capazes de fornecer recursos potenciais para favorecer a geração de novas alternativas e a adaptação (Lengnick-Hall et al., 2011).

Os dois últimos elementos contextuais de resiliência organizacional podem ser associados à característica "governança e parceria" atribuída às organizações híbridas. Essa associação é estabelecida sob a alegação de que essa característica salienta dimensões como a "prática de gestão participativa e o desenvolvimento de uma rede colaborativa", além de uma dependência da integração, interdependência e cooperação para o desenvolvimento da resiliência organizacional.

A existência de associações entre os elementos contextuais e as características singulares de organizações híbridas já havia sido abordada na análise do mapa conceitual. Essas associações agora estão mais claras e bem delimitadas. Uma síntese relatando essas associações pode ser visualizada através do diagrama representativo do modelo processual proposto na Figura 2. 


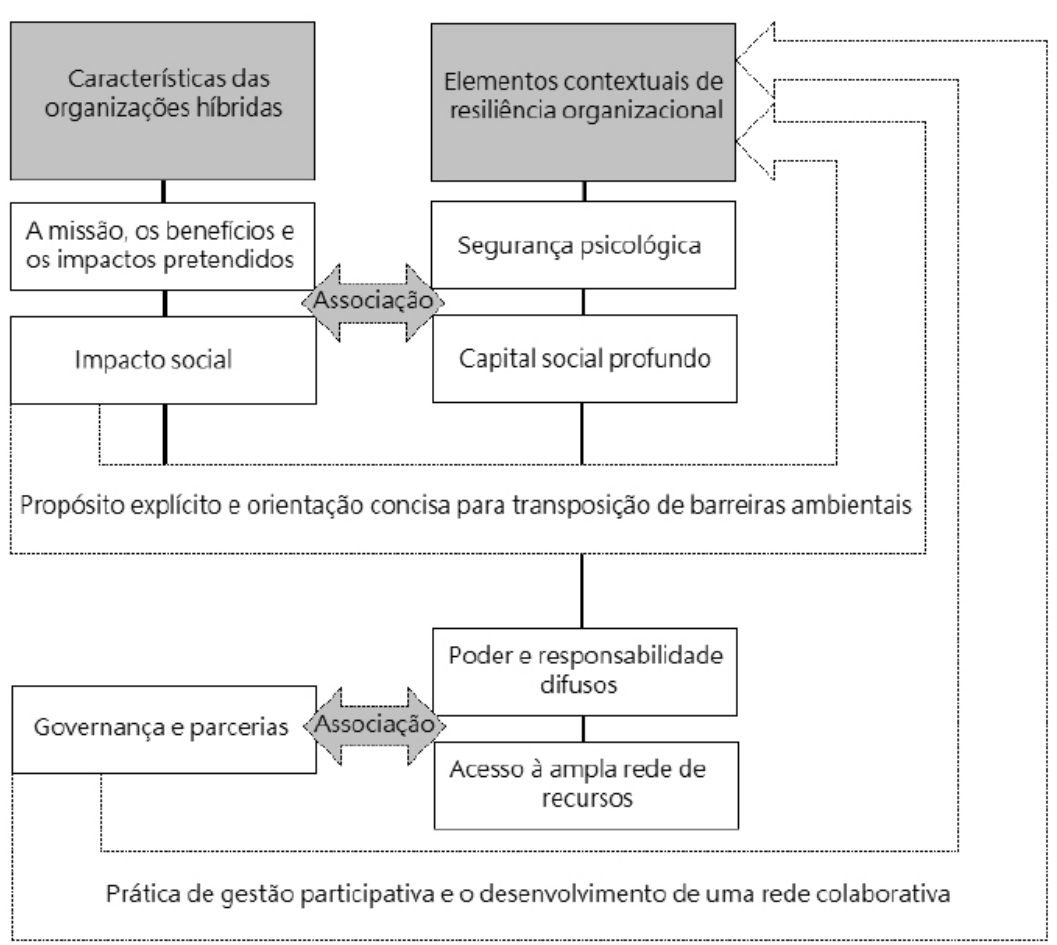

Figura 2. Modelo processual.

Fonte: Elaboração própria.

\section{Discussão e conclusões}

A identificação de características singulares de organizações híbridas que envolvem processos capazes de favorecer a resiliência organizacional foi a abordagem central deste estudo. Para alcançar esse objetivo, realizou-se uma revisão sistemática, na qual o interesse era investigar as organizações híbridas. Essa investigação permitiu que fossem elencadas características singulares de organizações híbridas, afinal, uma lista de características bem justificadas através da literatura é de grande valia para diversos pesquisadores subsidiarem seus estudos.

É importante observar que a amplitude da revisão não garante a abrangência da caracterização de organizações híbridas. O conjunto de características apresentado está em consonância com a literatura atual, mas essa caracterização pode ser influenciada futuramente através de novos cenários e situações nas quais as organizações híbridas estão envolvidas.

Através do procedimento de revisão, foi possível verificar que as características abordadas envolvem processos capazes de favorecer a resiliência organizacional. Também analisamos que essas características apresentam associações com dimensões de resiliência organizacional, mais especificamente, com elementos contextuais de resiliência organizacional.

A base central das ideias discutidas neste artigo é a proposição de um diálogo entre características de organizações híbridas e resiliência organizacional. As associações propostas revelam dimensões e processos de gestão provenientes de características com potencial para favorecer a 
construção da resiliência organizacional. Tal diálogo é capaz de motivar estudos por meio da visão de outros pesquisadores acerca desse mesmo tema.

As organizações híbridas desenvolvem habilidades que permitem conciliar lógicas concorrentes e o progresso dessas habilidades é alcançado porque as organizações híbridas são guiadas por um forte senso de propósito e valores. Estas, inteligência adaptativa que, aliada ao forte senso, fomentam internamente os potenciais transformacional e de cooperação. Esses potenciais geram oportunidade para um comportamento dinâmico e inovador, o qual resulta em estratégias multidisciplinares e sustentáveis úteis para responder aos diferentes desafios.

Vale ressaltar que tais habilidades permitem prosperar em ambientes adversos e desafiadores, sendo destacadas como importantes dimensões e processos de gestão para resiliência organizacional: (1) a função explícita de promover transformações positivas; (2) os modelos de negócios para a transposição de barreiras de transação; (3) o potencial para equacionar a oscilação de recursos tangíveis e as incompatibilidades com práticas vigentes na organização; (4) os valores que reforçam as práticas sustentáveis; (5) a capacidade de harmonizar e moldar processos de gestão; (6) a prática de gestão participativa e o desenvolvimento de redes colaborativas; (7) a gestão plural e estratégias inéditas; e, por fim, (8) as diversificadas configurações organizacionais.

As organizações híbridas buscam a conciliação de lógicas concorrentes e isso ocorre principalmente no âmbito das orientações relativas a sua missão dual (social/financeira). Lógicas relacionadas às práticas sociais visam promoção de impacto social positivo, enquanto as lógicas relacionadas às práticas de mercado visam objetivos financeiros. Logo, tratam-se de lógicas contraditórias, tanto de forma ontológica quanto de forma epistemológica. As lógicas que orientam às práticas de mercado visam lucro através de eficiência nas transações para a realização do melhor negócio e, tal formato, faz da maioria das lógicas relacionadas às práticas de mercado algo antagônico com respeito às lógicas relacionadas com práticas sociais. As premissas da realidade diária dessas organizações confirmam que esse antagonismo é relatado por pesquisadores da área.

As importantes dimensões e processos de gestão discutidos originam das características de organizações híbridas elencadas neste estudo, sendo também respaldados por meio dos elementos de resiliência organizacional.

A análise das características de organizações híbridas motivou uma exploração direcionada aos elementos contextuais da resiliência organizacional. A alegação central dessa exploração consiste no fato de que tais elementos configuram competências internas da organização (elementos contextuais da resiliência organizacional), que se constituem como um importante canal de interação externa em benefício de ações efetivas, de forma que a organização evolua e promova novas capacidades frente às complexidades do mercado.

Apresentou-se uma perspectiva deassociação dos elementos contextuais da resiliência organizacional com três características das organizações híbridas: (i) a missão, os benefícios, e os impactos pretendidos; (ii) impacto 
social; (iii) governança e parcerias. Essas características foram avaliadas como potenciais desenvolvedoras de dimensões capazes de contribuir para a resiliência organizacional. Dentre essas dimensões, evidenciou-se: um forte senso de propósito e valores fundamentais; integração; interdependência e cooperação. Essa associação é representada através do modelo processual proposto.

O modelo processual relata associações entre organizações híbridas e resiliência organizacional, mas não tem o propósito de cobrir integralmente a interseção entre organizações híbridas e resiliência organizacional. A relevância, nesse enquadramento, consiste na proposição de uma associação entre esses dois domínios. As informações apresentadas contribuem para a compreensão de dimensões que estimulam a postura de constante inquietação na busca por novas formas de atuação em prol da geração de valor social e sustentável.

Os elementos contextuais da resiliência organizacional representam competências internas da organização e propiciam a interação com agentes externos a favor de atitudes assertivas frente às condições ambientais adversas. A segurança psicológica e o capital social profundo podem ser associados às características das organizações híbridas "a missão, os benefícios, e os impactos pretendidos" e "impacto social". Já o poder e as responsabilidades difusas e o acesso à rede de recursos ampla se associam à característica "governança e parceria".

A limitação deste estudo é reconhecida em relação à abrangência das características eleitas, que é influenciada pelo cotidiano das organizações. Outra limitação diz respeito à aplicabilidade em outros contextos, como o de organizações voltadas exclusivamente às práticas de mercado.

O diálogo proposto e a perspectiva de associação das características de organizações híbridas com elementos contextuais da resiliência organizacional permitem a compreensão da articulação destes conceitos. Além disso, indicam algumas propostas de novas direções de continuidade de pesquisa, como a forma efetiva como algumas importantes dimensões e processos de gestão das organizações híbridas são destacados como potenciais contribuidores em prol da resiliência organizacional. Outra proposta de continuidade relevante seria discutir a necessidade de exames empíricos de dimensões e processos de gestão que apresentam maiores potenciais para favorecer o desenvolvimento de organizações resilientes.

De forma análoga, seria pertinente uma análise empírica para identificar características similares em outros tipos de organizações e as respectivas dimensões dessas características que são capazes de contribuir para a resiliência organizacional. Além disso, poderiam ser analisados os elementos cognitivos e comportamentais da resiliência organizacional de forma teórica e empírica em relação às características singulares de organizações híbridas apresentadas neste artigo. 


\section{Referências}

Abdullah, N. A. S., Noor, N. L. M., \& Ibrahim, E. N. M. (2013). Resilient organization: Modelling the capacity for resilience. Proceedings of the 2013 International Conference on Research and Innovation in Information Systems (ICRIIS) (pp. 319-324). Kuala Lumpur, KL, Malaysia, 3.

Alberti, F. G., \& Varon Garrido, M. A. (2017). Can profit and sustainability goals co-exist? New business models for hybrid firms. Journal of Business Strategy, 38(1), 3-13.

Alexius, S., \& Furusten, S. (2019). Enabling Sustainable Transformation: Hybrid Organizations in Early Phases of Path Generation. Journal of Business Ethics, (online) 1-17. DOI: 10.1007/s10551-018-04098-0

Almandoz, J., Lee, M., \& Marquis, C. (2017). Different shades of green: Environment uncertainty and the strategies of hybrid organizations. Research in the Sociology of Organizations, 50, 31-67.

Anheier, H. K., \& Krlev, G. (2015). Guest editors' introduction: Governance and management of hybrid organizations. International Studies of Management \& Organization, 45(3), 193-206.

Ávila, L., \& Amorim, M. (2017). Operational Challenges in Hybrid Organizations: Insights for Future Research. In L. Lou \& A. Worthington (Orgs.). Handbook of Research on Emerging Business Models and Managerial Strategies in the Nonprofit Sector (pp.352-370). Queensland: IGI Global.

Avey, J. B., Reichard, R. J., Luthans, F., \& Mhatre, K. H. (2011). Meta-analysis of the impact of positive psychological capital on employee attitudes, behaviors, and performance. Human Resource Development Quarterly, 22(2), 127-152.

Ayala, J. C., \& Manzano, G. (2014). The resilience of the entrepreneur. Influence on the success of the business. A longitudinal analysis. Journal of Economic Psychology, 42, 126-135.

Bandura, A. (1997), Self Efficacy: The Exercise of Control. New York: W. H. Freeman.

Barki, E., Izzo, D., Torres, H., \& Aguiar, L. (2013). Negócios com impacto social no Brasil. São Paulo: Editora Peirópolis.

Battilana, J. (2018). Cracking the organizational challenge of pursuing joint social and financial goals: Social enterprise as a laboratory to understand hybrid organizing. M@n@gement, 21(4), 1278-1305.

Battilana, J., \& Dorado, S. (2010). Building sustainable hybrid organizations: The case of commercial microfinance organizations. Academy of Management Journal, 53(6), 1419-1440.

Battilana, J., Besharov, M., \& Mitzinneck, B. (2017) On hybrids and hybrid organizing: A review and roadmap for future research. In R. Greenwood, C. Oliver, T. B. Lawrence \& R. E. Meyer (Eds.). The SAGE handbook of organizational institutionalism (pp. 133-169). London: Sage Publications.

Battilana, J., Lee, M., Walker, J., \& Dorsey, C. (2012). In search of the hybrid ideal. Stanford Social Innovation Review, 10(3), 50-55.

Battilana, J., \& Lee, M. (2014). Advancing research on hybrid organizing-Insights from the study of social enterprises. The Academy of Management Annals, 8(1), 397-441. 
Battilana, J., Sengul, M., Pache, A. C., \& Model, J. (2015). Harnessing productive tensions in hybrid organizations: The case of work integration social enterprises. Academy of Management Journal, 58(6), 1658-1685.

Bauwens, T., Huybrechts, B., \& Dufays, F. (2019). Understanding the diverse scaling strategies of social enterprises as hybrid organizations: The case of renewable energy cooperatives. Organization \& Environment, (online). DOI: 10.1177/1086026619837126

Bearman, M., \& Dawson, P. (2013). Qualitative synthesis and systematic review in health professions education. Medical Education, 47(3), 252-260.

Bergheim, K., Eid, J., Hystad, S. W., Nielsen, M. B., Mearns, K., Larsson, G., \& Luthans, B. (2013). The role of psychological capital in perception of safety climate among air traffic controllers. Journal of Leadership \& Organizational Studies, 20(2), 232-241.

Bobsin, D., Petrini, M., \& Pozzebon, M. (2019). The value of technology affordances to improve the management of nonprofit organizations. RAUSP Management Journal, 54(1), 14-37.

Braes, B., \& Brooks, D. (2011). Organizational Resilience: Understanding and identifying the essential concepts. Safety and Security Engineering, 4(117), 117-128.

Bullough, A., Renko, M., \& Myatt, T. (2014). Dangerzone entrepreneurs: The importance of resilience and self-efficacy for entrepreneurial intentions. Entrepreneurship Theory and Practice, 38(3), 473-499.

Burnes, B. (2004). Managing change: A strategic approach to organisational dynamics. London: Pearson Education.

Cañas, A. J., \& Novak, J. D. (2008). Concept mapping using CmapTools to enhance meaningful learning. In A. Osaka, S. B. Shum \& T. Sherborne (Eds.). Knowledge Cartography, Advanced Information and Knowledge Processing (pp. 25-46). Berlin: Springer Verlag.

Cañas, A. J., Novak, J. D., \& Reiska, P. (2015). How good is my concept map? Am I a good Cmapper? Knowledge Management \& E-Learning: An International Journal, 7(1), 6-19.

Chandra, Y., \& Shang, L. (2017). Unpacking the biographical antecedents of the emergence of social enterprises: A narrative perspective. VOLUNTAS: International Journal of Voluntary and Nonprofit Organizations, 28(6), 2498-2529.

Choi, N., \& Majumdar, S. (2014). Social entrepreneurship as an essentially contested concept: Opening a new avenue for systematic future research. Journal of Business Venturing, 29(3), 363-376.

Clarke M., \& Oxman A. D. (2001), Formulating the problem. Cochrane Reviewers' Handbook 4.1 In Review Manager (RevMan) [Computer program]. Version 4.1. Oxford, England: The Cochrane Collaboration.

Cooper, N., Estes, C. A., \& Allen, L. (2004). Bouncing back. Parks \& Recreation (Ashburn), 39(4), 28-35.

Creswell, J. W. (2013). Qualitative inquiry and research design: Choosing among five approaches. London: Sage Publications.

Coutu, D. L. (2002). How resilience works. Harvard Business Review, 80(5), 46-56. 
Danes, S. M., Lee, J., Amarapurkar, S., Stafford, K., Haynes, G., \& Brewton, K. E. (2009). Determinants of family business resilience after a natural disaster by gender of business owner. Journal of Developmental Entrepreneurship, 14(04), 333-354.

Dardour, A. (2012). Les modèles économiques en entrepreneuriat social: Proposition d'un modèle intégrateur. La Revue des Sciences de Gestion, 3, 49-57.

Dawson, P. (2005). Changing manufacturing practices: An appraisal of the processual approach. Human Factors and Ergonomics in Manufacturing \& Service Industries, 15(4), 385-402.

Doherty, B., Haugh, H., \& Lyon, F. (2014). Social enterprises as hybrid organizations: A review and research agenda. International Journal of Management Reviews, 16(4), 417-436.

Dufays, F., \& Huybrechts, B. (2016). Where do hybrids come from? Entrepreneurial team heterogeneity as an avenue for the emergence of hybrid organizations. International Small Business Journal, 34(6), 777-796.

Ebrahim, A., Battilana, J., \& Mair, J. (2014). The governance of social enterprises: Mission drift and accountability challenges in hybrid organizations. Research in Organizational Behavior, 34, 81-100.

Fink, A. (2005). Conducting Research Literature Reviews: From the Internet to Paper (2nd ed.). Thousand Oaks, California: Sage Publications.

Gibson, C. A., \& Tarrant, M. (2010). A 'conceptual models' approach to organisational resilience. The Australian Journal of Emergency Management, 25(2), 6.

Gittell, J. H., Cameron, K., Lim, S., \& Rivas, V. (2006). Relationships, layoffs, and organizational resilience: Airline industry responses to September 11. The Journal of Applied Behavioral Science, 2(3), 300-329.

Grossi, G., \& Thomasson, A. (2015). Bridging the accountability gap in hybrid organizations: the case of Copenhagen Malmö Port. International Review of Administrative Sciences, 81(3), 604-620.

Gupta, S., Beninger, S., \& Ganesh, J. (2015). A hybrid approach to innovation by social enterprises: lessons from Africa. Social Enterprise Journal, 11(1), 89-112.

Haigh, N., Walker, J., Bacq, S., \& Kickul, J. (2015). Hybrid organizations: origins, strategies, impacts, and implications. California Management Review, 57(3), 5-12.

Hahn, R., \& Ince, I. (2016). Constituents and characteristics of hybrid businesses: A qualitative, empirical framework. Journal of Small Business Management, 54(S1), 33-52.

Hamel, G., \& Valikangas, L. (2004). The quest for resilience. Revista Icade Revista de las Facultades de Derecho y Ciencias Económicas y Empresariales, 62, 355-358.

Jäger, U. P., \& Schröer, A. (2014). Integrated organizational identity: A definition of hybrid organizations and a research agenda. VOLUNTAS: International Journal of Voluntary and Nonprofit Organizations, 25(5), 1281-1306.

Jay, J. (2013). Navigating paradox as a mechanism of change and innovation in hybrid organizations. Academy of Management Journal, 56(1), 137-159.

Johansen, S. T., Olsen, T. H., Solstad, E., \& Torsteinsen, H. (2015). An insider view of the hybrid organisation: How managers respond to challenges of efficiency, legitimacy and meaning. Journal of Management \& Organization, 21(6), 725-740. 
Kannothra, C. G., Manning, S., \& Haigh, N. (2018). How hybrids manage growth and social-business tensions in global supply chains: The case of impact sourcing. Journal of Business Ethics, 148(2), 271-290.

Kendra, J. M., \& Wachtendorf, T. (2003). Elements of community resilience in the World Trade Center attack. Disasters, 27(1), 37-53.

Kolk, A., \& Lenfant, F. (2016). Hybrid business models for peace and reconciliation. Business Horizons, 59(5), 503-524.

Koppell, J. G. (2003) The Politics of Quasi-Government. New York: Cambridge University Press.

Korber, S., \& McNaughton, R. B. (2018). Resilience and entrepreneurship: a systematic literature review. International Journal of Entrepreneurial Behavior \& Research, 24(7), 1129-1154.

Kroeger, A., \& Weber, C. (2014). Developing a conceptual framework for comparing social value creation. Academy of Management Review, 39(4), 513-540.

Krueger, A., \& Behringer, J. (2017). Hybrid Organising in Social Entrepreneurship: How Do Founders of Social Ventures Experience the Tensions in the Areas of Acquiring and Allocating Resources? (Student papers) Disponivel via Lund University em: http://lup.lub.lu.se/student-papers/record/8914363

Lampel, J., Bhalla, A., \& Jha, P. P. (2014). Does governance confer organizational resilience? Evidence from UK employee owned businesses. European Management Journal, 32(1), 66-72.

Lee, A. V., Vargo, J., \& Seville, E. (2013). Developing a tool to measure and compare organizations' resilience. Natural Hazards Review, 14(1), 29-41.

Lee, M., \& Jay, J. (2015). Strategic responses to hybrid social ventures. California Management Review, 57(3), 126-147.

Lengnick-Hall, C. A., \& Beck, T. E. (2003). Beyond bouncing back: The concept of organizational resilience. Proceedings of the National Academy of Management meetings, Seattle, WA, USA, 80.

Lengnick-Hall, C. A., \& Beck, T.E. (2005). Adaptive fit versus robust transformation: How organizations respond to Environmental Change. Journal of Management, 3(5)1, 738-757.

Lengnick-Hall, C. A., \& Beck, T. E. (2016). Resilience capacity and strategic agility: Prerequisites for thriving in a dynamic environment. In E. Hollnagel, C. P. Nemeth \& S. Dekker (Eds.). Resilience Engineering Perspectives, (Vol 2, pp. 61-92). Boca Raton: CRC Press.

Lengnick-Hall, C. A., Beck, T. E., \& Lengnick-Hall, M. L. (2011). Developing a capacity for organizational resilience through strategic human resource management. Human Resource Management Review, 21(3), 243-255.

Lepoutre, J., Justo, R., Terjesen, S., \& Bosma, N. (2013). Designing a global standardized methodology for measuring social entrepreneurship activity: The Global Entrepreneurship Monitor social entrepreneurship study. Small Business Economics, 40(3), 693-714.

Limnios, E. A. M., Mazzarol, T., Ghadouani, A., \& Schilizzi, S. G. (2014). The resilience architecture framework: four organizational archetypes. European Management Journal, 32(1), 104-116. 
Linnenluecke, M. K. (2017). Resilience in business and management research: A review of influential publications and a research agenda. International Journal of Management Reviews, 19(1), 4-30.

Luthans, F. (2002). The need for and meaning of positive organizational behavior. Journal of Organizational Behavior, 23(6), 695-706.

Luthans, B. C., Luthans, K. W., \& Avey, J. B. (2014). Building the leaders of tomorrow: The development of academic psychological capital. Journal of Leadership \& Organizational Studies, 21(2), 191-199.

Mallak, L. (1998). Putting organizational resilience to work. Industrial Management, 40(6), 8-13.

Mair, J., Mayer, J., \& Lutz, E. (2015). Navigating institutional plurality: Organizational governance in hybrid organizations. Organization Studies, 36(6), 713-739.

Manning, S., Kannothra, C. G., \& Wissman-Weber, N. K. (2017). The Strategic Potential of Community-Based Hybrid Models: The Case of Global Business Services in Africa. Global Strategy Journal, 7(1), 125-149.

Martin, R. L., \& Osberg, S. (2007). Social entrepreneurship: The case for definition. Stanford Social Innovation Review, 5(2), 28-39.

McManus, S., Seville, E., Brunsdon, D., \& Vargo, J. (2007). Resilience management: a framework for assessing and improving the resilience of organizations (Research Report 2007/01), Christchurch, New Zealand, Resilient Organisations Research Group, University of Canterbury. Department of Accounting and Information Systems.

McMullen, J. S. (2018). Organizational hybrids as biological hybrids: Insights for research on the relationship between social enterprise and the entrepreneurial ecosystem. Journal of Business Venturing, 33(5), 575-590.

McMullen, J. S., \& Warnick, B. J. (2016). Should we require every new venture to be a hybrid organization? Journal of Management Studies, 53(4), 630-662.

Memili, E., Welsh, D. H., \& Luthans, F. (2013). Going beyond research on goal setting: A proposed role for organizational psychological capital of family firms. Entrepreneurship Theory and Practice, 37(6), 1289-1296.

Meyer, A. D. (1982). Adapting to environmental jolts. Administrative Science Quarterly, 27(4), 515-537.

Miller, T. L., Grimes, M. G., McMullen, J. S., \& Vogus, T. J. (2012). Venturing for others with heart and head: How compassion encourages social entrepreneurship. Academy of Management Review, 37(4), 616-640.

Mitronen, L., \& Möller, K. (2003). Management of hybrid organizations: a case study in retailing. Industrial Marketing Management, 32(5), 419-429.

Mongelli, L., \& Rullani, F. (2017). Inequality and marginalisation: social innovation, social entrepreneurship and business model innovation: The common thread of the DRUID Summer Conference 2015. Industry and Innovation, 24(5), 446-467.

Mongelli, L., Rullani, F., \& Versari, P. (2017). Hybridization of diverging institutional logics through common-note practices-an analogy with music and the case of social enterprises. Industry and Innovation, 24(5), 492-514.

Moss, T. W., Renko, M., Block, E., \& Meyskens, M. (2018). Funding the story of hybrid ventures: Crowdfunder lending preferences and linguistic hybridity. Journal of Business Venturing, 33(5), 643-659. 
Novak, J. D. (1990). Concept mapping: A useful tool for science education. Journal of Research in Science Teaching, 27(10), 937-949.

Okoli, C., \& Schabram, K. (2010). A Guide to Conducting a Systematic Literature Review of Information Systems Research. SSRN Electronic Journal, 10. DOI: 10.2139/ssrn.1954824

Pache, A. C. (2010). The art of managing conflicting institutional logics: The case of social integration enterprises. Academy of Management Proceeding, 2010(1), 1-6.

Pache, A. C., \& Santos, F. (2010). When worlds collide: The internal dynamics of organizational responses to conflicting institutional demands. Academy of Management Review, 35(3), 455-476.

Pache, A. C., \& Santos, F. (2013). Inside the hybrid organization: Selective coupling as a response to competing institutional logics. Academy of Management Journal, 56(4), 972-1001.

Park, J. H. (2019). Chasing two rabbits: how social enterprises as hybrid organizations manage paradoxes. Asian Business \& Management, 1-31.

Parra, S. (2013). Exploring the incorporation of values for sustainable entrepreneurship teaching/learning. Journal of Technology Management \& Innovation, 8(1), 11-20.

Pettigrew, A. M., Woodman, R. W., \& Cameron, K. S. (2001). Studying organizational change and development: Challenges for future research. Academy of Management Journal, 44(4), 697-713.

Plotnick, E. (1997). Concept mapping: A graphical system for understanding the relationship between concept. Syracuse, NY: ERIC Clearinghouse on Information and Technology.

Quinn, Q. C., \& Munir, K. A. (2017). Hybrid categories as political devices: The case of impact investing in frontier markets. In R. Durrand, N. Granqvist, A. Tyllström (Eds.). From Categories to Categorization: Studies in Sociology, Organizations and Strategy at the Crossroads (pp. 113-150). Bingley: Emerald Publishing Limited.

Reissner, S. C. (2019). 'We are this hybrid': Members' search for organizational identity in an institutionalized public-private partnership. Public Administration, 97(1), 48-63.

Rodríguez-Sánchez, A. M., \& Perea, M. V. (2015). The secret of organization success: a revision on organisational and team resilience. International Journal of Emergency Services, 4(1), 27-36.

Roundy, P. T. (2017). Hybrid organizations and the logics of entrepreneurial ecosystems. International Entrepreneurship and Management Journal, 13(4), 1221-1237.

Santos, F., Pache, A. C., \& Birkholz, C. (2015). Making hybrids work: Aligning business models and organizational design for social enterprises. California Management Review, 57(3), 36-58.

Schildt, H., \& Perkmann, M. (2017). Organizational settlements: theorizing how organizations respond to institutional complexity. Journal of Management Inquiry, 26(2), 139-145.

Schmitz, B. (2015). Beyond structural governance: Tension moments and the preservation of core values in hybrid organizations. International Studies of Management \& Organization, 45(3), 241-258. 
Shin, J., Taylor, M. S., \& Seo, M. G. (2012). Resources for change: The relationships of organizational inducements and psychological resilience to employees' attitudes and behaviors toward organizational change. Academy of Management Journal, 55(3), 727-748.

Somers, S. (2009). Measuring resilience potential: An adaptive strategy for organizational crisis planning. Journal of Contingencies and Crisis Management, 17(1), 12-23.

Sutcliffe, K. M., \& Vogus, T. J. (2003). Organizing for resilience. In K. S. Cameron, J. E. Dutton \& R. E. Quinn (Eds.). Positive organizational scholarship: Foundations of a new discipline, 94(1), 110.

Tedeschi, R. G., \& Calhoun, L. G. (2004). Target Article: "Posttraumatic Growth: Conceptual Foundations and Empirical Evidence". Psychological Inquiry, 15(1), 1-18.

Treinta, F. T., Farias Filho, J. R., Sant'Anna, A. P., \& Rabelo, L. M. (2014). Metodologia de pesquisa bibliográfica com a utilização de método multicritério de apoio à decisão. Production, 24(3), 508-520.

Uman, L. S. (2011) Systematic reviews and meta-analyses. Journal of the Canadian Academy of Child and Adolescent Psychiatry, 20(1), 57-59.

Vogus, T. J., \& Sutcliffe, K. M. (2007, October). Organizational resilience: towards a theory and research agenda. Conference Proceedings - IEEE Systems, Man and Cybernetics, Montréal, Canada, 7-10.

Webster, J., \& Watson, R. T. (2002). Analyzing the past to prepare for the future: Writing a literature review. MIS quarterly, 26(2), xiii-xxiii.

Weick, K. E., \& Sutcliffe, K. M. (2011). Managing the unexpected: Resilient performance in an age of uncertainty (Vol.8). San Francisco: John Wiley \& Sons.

Wilson, F., \& Post, J. E. (2013). Business models for people, planet (\& profits): Exploring the phenomena of social business, a market-based approach to social value creation. Small Business Economics, 40(3), 715-737.

Windle, G., Bennett, K. M., \& Noyes, J. A. (2011). Methodological review of resilience measurement scales. Health and Quality of Life Outcomes, 9(1), 8.

Wolf, M., \& Mair, J. (2019) Purpose, Commitment and Coordination Around Small Wins: A Proactive Approach to Governance in Integrated Hybrid Organizations. VOLUNTAS: International Journal of Voluntary and Nonprofit Organizations, 30(3), 535-548.

Wood Jr., T. (2010). Organizações híbridas. Revista de Administração de Empresas, 50(2), 241-247.

Woods, D. D., \& Wreathall, J. (2008). Stress-strain plots as a basis for assessing system resilience. Resilience Engineering Perspectives, 1, 145-161.

York, J. G., Hargrave, T. J., \& Pacheco, D. F. (2016a). Converging winds: Logic hybridization in the Colorado wind energy field. Academy of Management Journal, 59(2), 579-610.

York, J. G., O'Neil, I., \& Sarasvathy, S. D. (2016b). Exploring environmental entrepreneurship: identity coupling, venture goals, and stakeholder incentives. Journal of Management Studies, 53(5), 695-737.

Zahra, S. A., \& Wright, M. (2016). Understanding the social role of entrepreneurship. Journal of Management Studies, 53(4), 610-629. 